4. Which of the following is true for the Schirmer tear test in cats?

a. Is difficult to interpret due to poor repeatability and wide normal variability

b. Should be performed after application of a topical anaesthetic

c. Should be performed in the ventromedial conjunctival fornix d. Has a median (and range) in normal cats of $5(0-16) \mathrm{mm} / \mathrm{min}$

5. Which of the following is true for fluorescein staining?

a. Should be performed before the STT

b. Is of no value in eyes with normal IOP

c. Is necessary only when corneal changes are noted during the examination

d. If correctly performed does not need flushing

\section{FHV-1 and cat corneas: are you up to date?}

\section{David Maggs}

Feline herpesvirus (FHV-1) is a ubiquitous virus with relatively consistent virulence worldwide and, yet, we see a huge range of clinical signs in infected cats. The most likely reason for this is the variable host response to this virus. Naiive kittens infected against a backdrop of waning maternal immunity almost inevitably get severe upper respiratory and bilateral ocular disease with high morbidity but rare mortality. By contrast, adult cats can undergo viral reactivation with viral shedding that can infect in-contact cats but without demonstrating clinical signs themselves, or can develop debilitating but often unilateral ocular, respiratory or dermatological syndromes. These scenarios demonstrate the broad diversity of clinical signs seen with $\mathrm{FHV}-1$ in clinical practice. An understanding that normal cats frequently shed virus also explains why virological testing is of little diagnostic value.

Although FHV-1 preferentially infects conjunctival cells, corneal disease (either through direct herpetic infection or as a consequence of marked conjunctivitis) is particularly important because it is painful, often not completely reversible and potentially blinding. The pathognomonic sign of corneal herpetic disease is dendritic ulcers; however these are often missed as they are transient and occur early following infection or reactivation before rapidly coalescing to form geographic ulcers. If conjunctival ulceration occurs concurrently, symblepharon formation is possible. Although recrudescent disease occurs in a minority of latently infected cats, it produces serious corneal stromal and epithelial diseases which may be immunopathological (i.e. immune-mediated, but not necessarily autoimmune - such as herpetic stromal keratitis or eosinophilic keratitis), cytolytic (i.e. ulcerative) or metaherpetic (i.e. resulting from structural tissue damage subsequent to cytolytic or immunopathological disease). The concept of metaherpetic disease is relatively new in veterinary medicine and is exemplified by tear film deficiency secondary to herpetic damage of lacrimal nerves or ablation of conjunctival goblet cells responsible for tear mucins. Determining which of these three pathogenic mechanisms is likely is critical for selection of effective therapies.

Therapy for herpetic disease can be supportive (such as antibiotics for treatment or prophylaxis of secondary infection, tear substitutes for dry eye, lysine as an adjunctive therapy that may limit viral replication, or the interferons which alter the immune state to be more resistant to viruses) or topically or systemically administered antiviral agents (such as famciclovir, cidofovir, ganciclovir, idoxuridine, trifluridine or aciclovir). The following are some important updates about these therapies:

Lysine should be given twice daily (500 mg orally) and NOT applied to food such that cats 'graze' it throughout the day

- There is minimal peer-reviewed evidence to support systemic or topical use of the interferons

- Famciclovir - a prodrug of penciclovir - is one of the best studied antiviral agents in cats, and when given at $90 \mathrm{mg} / \mathrm{kg}$ orally q12h creates effective tear penciclovir concentrations. Do not taper dose!

- All antiviral drugs are virostatic; therefore they are ineffective against latent virus and must be given very frequently to be effective

- FHV-1 causes long-term ablation of the conjunctival goblet cells that is not prevented or corrected with famciclovir. Topical hyaluronate may aid regeneration of conjunctival goblet cells and is an essential adjunctive therapy in cats with herpetic disease

\section{KEY LEARNING OBJECTIVES}

- To apply some basic virology in the diagnosis and treatment of FHV-1 in cats

- To understand the limitations of virostatic drugs and how these are minimised by therapeutic choices

- To understand the importance of tear film dysfunction and augmentation in feline herpetic disease

\section{MULTIPLE CHOICE QUESTIONS}

1. Which of the following statements about feline herpesvirus infection in cats is true?

a. Rarely establishes latency

b. Recurs throughout life with similar bilateral signs to those seen at first infection

c. Typically causes severe bilateral signs in cats infected for the first time

d. Is typically recurrent only during the first 2-3 years after primary infection

2. Which of the following statements about feline herpesvirus infection in cats is true?

a. Always produces ulcerative corneal disease

b. Has been associated with feline eosinophilic keratitis

c. Is not associated with corneal sequestrum development

d. Should be treated topically 


\section{Frightened of feline ophthalmology}

3. Which of the following statements about antiviral drugs currently available for treatment of cats with $\mathrm{FHV}-1$ is true?
a. Are all virostatic
b. Are available only in topical formulations
c. Are typically given once to twice daily
d. Are effective against latent virus

4. Which of the following statements about famciclovir is true?
a. Is virucidal
b. Is a prodrug of penciclovir
c. Is not appropriate for corneal disease because it is

an oral medication

d. Should be tapered (with regards dose or dose frequency) as signs improve

5. Which of the following statements about lysine is true?

a. Is a highly potent antiviral drug

b. Should be given as a dietary supplement to avoid pilling

c. Has not been studied for its effect in cats with FHV-1

d. May be of some adjunctive value in the treatment of FHV-1 when administered at 500 mg orally q12h

\section{Corneal trauma in cats: can I manage this myself?}

\section{Claudia Hartley}

Corneal trauma may be mechanical, blunt, penetrating, perforating, thermal or chemical. The trauma may also be acute (e.g. as a result of a cat fight) or chronic (e.g. as a result of entropion). Corneal trauma is frequently associated with a reflex uveitis, and if the trauma is penetrating, hyphaema may also be present. In this presentation we will discuss the causes and diagnosis of corneal trauma in cats, how to approach treatment and which cases may require specialist care.

Mechanical trauma, from lagophthalmia, entropion, ectropion (usually cicatricial in cats), eyelid agenesis, distichiasis or foreign material entrapment, can all result in corneal trauma.

Blunt corneal trauma may be more devastating to vision than sharp trauma due to the contrecoup effects on the globe, specifically the lens and retina, causing visionthreatening injuries. Where intraocular examination cannot be completed due to the opacity of the ocular media (cornea, aqueous, lens or vitreous), ocular ultrasonography should be undertaken.

Sharp trauma may cause injuries of varying depths, and may result in the entrapment of foreign material within the cornea or anterior chamber. Foreign material lodged in the conjunctiva (particularly on the posterior surface of the eyelid) may cause chronic corneal trauma until it is identified and removed.

Bacterial or fungal contamination of the cornea, or intraocular compartments if it is a penetrating injury, may complicate healing and requires appropriate treatment. Where lens trauma has occurred in conjunction with corneal trauma, it usually requires prompt surgery to remove the lens (and antigenic source) to avoid catastrophic phacoclastic uveitis.

Chemical trauma may be caused by contact with acidic or alkaline substances. In general, acidic substances cause coagulation of corneal collagen that limits further penetration of the acid. Alkaline substances, in contrast, tend to penetrate more deeply and result in greater damage to the cornea and intraocular structures. Loss of corneal epithelial stem cell populations that reside in the palisades of Voigt at the limbus, can have significant implications on healing of corneal ulceration, leading to chronic ulceration and/or conjunctivalisation of the corneal surface (symblepharon).

\section{KEY LEARNING OBJECTIVES}

- Recognise and investigate feline corneal trauma cases appropriately

- Identify appropriate emergency care for trauma cases

- Identify cases that require urgent referral

\section{MULTIPLE CHOICE QUESTIONS}

1. An owner presents her cat with a history of caustic soda splash to the left eye. What would be your first emergency treatment?
a. Apply an ocular lubricant
b. Sedate the cat to place a temporary tarsorrhaphy
c. Copiously flush the eye with saline
d. Apply an antibiotic ointment

2. When removing a partially embedded thorn corneal foreign body, under general anaesthesia and an operating microscope, how should you remove the thorn?
a. By grasping the thorn with fine rat-tooth forceps and pulling it out
b. By impaling it perpendicularly and extracting in the opposite direction it entered
c. By grasping the thorn with fine rat-tooth forceps and rotating it outwards in a spiral
d. By impaling it perpendicularly and rotating it outwards in a spiral

3. What is a cat with chronic untreated entropion at risk of developing?
a. Sequestrum
b. Cataract
c. Retinal detachment
d. Glaucoma

4. With what should corneal malacia following corneal trauma be treated?
a. Topical steroids
b. Topical serum
c. Topical non-steroidal anti-inflammatory drug (NSAID)
d. Topical lubricant 